\title{
168. The Isometry Groups of Compact Manifolds with Non-positive Curvature*)
}

\author{
By Masao MAedA \\ Department of Mathematics, Tokyo Institute of Technology \\ (Comm. by Kinjirô KUNUGI, M. J. A., Oct. 13, 1975)
}

Let $M$ be an $n$-dimensional compact connected Riemannian manifold with negative Ricci curvature. Then a classical theorem of Bochner says that there exist no non-trivial Killing vector fields on $M$. And hence the order of the isometry group $I(M)$ of $M$ is finite. Relating to this theorem, T. Frankel obtained the following:

Let $M$ be a compact Riemannian manifold with non-positive sectional curvature $K_{\sigma} \leqq 0$ and with negative Ricci curvature. If $f: M \rightarrow M$ is an isometry which is continuously homotopic to the identity map, then $f$ is the identity, see [1]. This result was extended by H.B. Lawson and S. T. Yau in a more general situation. That is

Theorem ([5; Theorem 4, p. 225]). Let M be a compact Riemannian manifold with non-positive sectional curvature and Ricci curvature negative at some point of $M$. If $f: M \rightarrow M$ is an isometry continuously homotopic to the identity, then $f$ is the identity.

As a corollary of this theorem, we easily have

Lemma 1. Let $M$ be a manifold as in the theorem of Lawson and Yau. If $f: M \rightarrow M$ is an isometry such that $d(p, f(p))<d(p, C(p))$ for all point $p \in M$, then $f$ is the identity.

Here $d$ is the distance function of $M$ induced from the Riemannian metric and $C(p)$ the cut locus of $p$ in $M$.

Now, for such manifolds as in the theorem of Bochner or Lawson and $\mathrm{Yau}$, it is natural to ask whether we can estimate the order of the isometry group $I(M)$ by using the geometrical terms of $M$, for example, the diameter, the injectivity radius, the sectional curvature and so on. To this problem, H. C. Im Hof gave an estimation of order of $I(M)$ for a manifold with the sectional curvature $K_{\sigma}$ satisfying $-b^{2} \leqq K_{o} \leqq-a^{2}<0$, $0<a \leqq b$. In his argument, the assumption that $M$ is of negative curvature is essential.

In this note, we will give an estimation of the order of $I(M)$ for manifolds as in the theorem of Lawson and Yau in a different way from the one in H. C. Im Hof's theorem. The author thanks Prof. T. Otsuki for his kind advices.

Let $M$ be a compact Riemannian manifold. For a point $p \in M$,

*) Dedicated to Prof. S. Kashiwabara on his 60th birthday. 
$T_{p}(M)$ denotes the tangent space of $M$ at $p$ and $\exp _{p}: T_{p}(M) \rightarrow M$ the exponential mapping. Every geodesic is parametrized with respect to the arc-length. Put $\delta:=\min \{d(p, C(p)): p \in M\} . \quad \delta$ is called the injectivity radius of $M$. For a positive number $r$ and $a$ point $p \in M, B_{r}(p)$ denotes the open metric ball in $M$ with radius $r$ centerd at $p$. By the compactness of $M$, we can choose a finite number of points $p_{1}, \cdots, p_{k} \in M$ such that

$$
\bigcup_{i=1}^{k} B_{\delta / 4}\left(p_{i}\right)=M
$$

Of course $k$ depend on $\delta$ and other geometrical structure of $M$. In this situation, we have

Proposition 1. Let $M$ be a compact Riemannian manifold with non-positive sectional curvature and Ricci curvature negative at some point of $M$. Then

$$
\text { the order of } I(M) \leqq k^{k} \text {. }
$$

Proof. We define a mapping $\Phi: I(M) \rightarrow$ all the $k$-sequences $\left(i_{1}, i_{2}\right.$, $\left.\left.\cdots, i_{k}\right), 1 \leqq i_{1}, i_{2}, \cdots, i_{k} \leqq k\right\}$ as follows : for $f \in I(M)$

$$
\Phi(f)=\left(j_{1}, j_{2}, \cdots, j_{k}\right)
$$

where for each $i=1, \cdots, k$, the number $j_{i}$ is the smallest of $j$ such that $f\left(p_{i}\right) \in B_{\delta / 4}\left(p_{j}\right) . \quad \Phi$ is well defined, because by means of $(*)$. We will show that $\Phi$ is injective. Assume $\Phi(f)=\Phi(g)=\left(j_{1}, j_{2}, \cdots, j_{k}\right)$ for $f, g$ $\in I(M)$. Then we have

$$
d\left(f\left(p_{i}\right), p_{j_{i}}\right)<\frac{\delta}{4} \quad \text { and } \quad d\left(g\left(p_{i}\right), p_{j_{i}}\right)<\frac{\delta}{4}, \quad i=1, \cdots, k .
$$

Hence, putting $h:=f^{-1} \circ g$, for each $i=1, \cdots, k$, we have

$$
\begin{aligned}
d\left(p_{i}, h\left(p_{i}\right)\right) & =d\left(p_{i}, f^{-1} \circ g\left(p_{i}\right)\right)=d\left(f\left(p_{i}\right), g\left(p_{i}\right)\right) \\
& \leqq d\left(f\left(p_{i}\right), p_{j_{i}}\right)+d\left(p_{j_{i}}, g\left(p_{i}\right)\right)<\frac{\delta}{2} .
\end{aligned}
$$

Now for each point $p \in M$, we can find a number $i_{0}$ such that $p \in B_{\delta / 4}\left(p_{i_{0}}\right)$ by $(*)$. So, from the above fact, we have

$$
\begin{aligned}
d(p, h(p)) & \leqq d\left(p, p_{i_{0}}\right)+d\left(p_{i_{0}}, h\left(p_{i_{0}}\right)\right)+d\left(h\left(p_{i_{0}}\right), h(p)\right) \\
& <\frac{\delta}{4}+\frac{\delta}{2}+d\left(p_{i_{0}}, p\right)<\frac{\delta}{4}+\frac{\delta}{2}+\frac{\delta}{4}=\delta .
\end{aligned}
$$

Thus for each point $p \in M, d(p, h(p))<\delta \leqq d(p, C(p))$. Then by Lemma $1, h$ must be identity. So our assertion is proved.

q.e.d.

Next, we will calculate the number $k$ for compact manifolds with the sectional curvature satisfying $-b^{2} \leqq K_{o} \leqq 0$ for some $b>0$.

Let $H$ be the $n$-dimensional hyperbolic space of constant curvature $-b^{2}$ represented on the Euclidean disk $\left\{x \in R^{n}:\|x\|^{2}<1 / b^{2}\right\}$ with the Riemannian metric $d s^{2}=\frac{4}{\left(1-b^{2}\|x\|^{2}\right)^{2}} \sum_{i=1}^{n} d x_{i}^{2}$. For a positive number $t$, [ $t$ ] denotes the integer part of $t$. Let $r, s, r>s$ be any positive numbers. 
Lemma 2. For the point $\bar{p}=(0, \cdots, 0) \in H$, there exists a finite number of points $\bar{p}_{1}, \cdots, \bar{p}_{l} \in B_{r}(\bar{p})$ such that

The number $l$ can be taken as

$$
\bigcup_{i=1}^{l} B_{s}\left(\bar{p}_{i}\right) \supset \overline{B_{r}(\bar{p})} \text {. }
$$

$$
l \leqq\left(\left[\frac{r}{s}\right]+1\right)\left(\left[\frac{2 R}{\alpha}\right]+1\right)^{n}+1
$$

where $R$ and $\alpha$ are detemined with $\beta$ and $\alpha$ as follows:

$$
\left\{\begin{array}{l}
\cosh b \beta=\cosh b s / \cosh \frac{b s}{2}, \quad a=\tanh \frac{b r}{2} / b, \\
R=2 a /\left(1-a^{2} b^{2}\right), \quad \alpha=\left\{\frac{2 R^{2}}{n}\left(1-\cos \frac{\beta}{R}\right)\right\}^{1 / 2} .
\end{array}\right.
$$

Proof. Let $\gamma:[0, \infty) \rightarrow H$ be a geodesic starting from $\bar{p}$. Consider a covering of $\gamma([0, r])$ :

$$
\bigcup_{j=0}^{[r / s]+1} B_{s}(\gamma(j \cdot s)) \supset \gamma([0, r]) .
$$

Let $\beta$ be the number satisfying $(* *)$. Then, by using the hyperbolic trigonometry, we can see that $\beta$-tubular neighborhood

$$
U_{\beta}(\gamma([0, r])):=\{\bar{q} \in H: d(\bar{q}, \gamma([0, r]))<\beta\}
$$

of $\gamma$ is contained in $\bigcup_{j} B_{s}(\gamma(j \cdot s))$. And we have

$$
U_{\beta}(\gamma([0, r])) \cap \partial B_{r}(\bar{p}) \supset B_{\beta^{\prime}}^{n-1}(\gamma(r)) \supset B_{\beta}^{n-1}(\gamma(r))
$$

for some $\beta^{\prime}>\beta$, where $B_{\beta^{\prime}}^{n-1}(\gamma(r))$ denotes the open metric ball in $\partial B_{r}(\bar{p})$ with radius $\beta^{\prime}$ centered at $\gamma(r)$. Note that $\partial B_{r}(\bar{p})$ is isometric to the standard ( $n-1)$-dimensional sphere $S^{n-1}(R)$. For any point $\bar{q} \in B_{\beta}^{n-1}(\gamma(r))$ and the geodesic $\eta:[0, r] \rightarrow H$ connecting from $\gamma(0)$ to $\bar{q}$, we easily see that $\eta([0, r]) \subset U_{\beta}(\gamma([0, r]))$. Thus, if we can find a finite number of points $\bar{p}_{0}, \cdots, \bar{p}_{m} \in S^{n-1}(R)$ such that

$$
\bigcup_{i=1}^{m} B_{\beta^{\prime}}^{n-1}\left(\bar{p}_{i}\right) \supset S^{n-1}(R),
$$

then, from the above fact, we have

$$
\overline{B_{r}(\bar{p})} \subset \bigcup_{i=1}^{m} U_{\beta}\left(\gamma_{i}([0, r])\right) \subset \bigcup_{i=1}^{m} \bigcup_{j=0}^{[r / s]+1} B_{s}\left(\gamma_{i}(j \cdot s)\right),
$$

where $\gamma_{i}:[0, r] \rightarrow H$ is the geodesic from $\bar{p}$ to $\bar{p}_{i}, i=1, \cdots, m$.

Now, for any point $p=\left(p_{1}, \cdots, p_{n}\right) \in R^{n}$ and a positive number $\rho>0$, we set

$$
I(p, \rho):=\left\{x=\left(x_{1}, \cdots, x_{n}\right) \in R^{n}: 0<\left|x_{i}-p_{i}\right|<\rho / 2,1 \leqq i \leqq n\right\} .
$$

Then we can easily see

$$
\begin{aligned}
& \sup \left\{d\left(q, q^{\prime}\right): q, q^{\prime} \in I(p, \rho) \cap S^{n-1}(R)\right\} \\
& \quad \leqq R \arccos \frac{2 R^{2}-n \rho^{2}}{2 R^{2}} \quad \text { if } n \rho^{2} \leqq 2 R^{2} .
\end{aligned}
$$

where $d$ is the distance function on $S^{n-1}(R)$. We choose $\alpha>0$ such that

$$
R \arccos \frac{2 R^{2}-n \alpha^{2}}{2 R^{2}}=\beta
$$


i.e. $\alpha=\left\{\frac{2 R^{2}}{n}\left(1-\cos \frac{\beta}{R}\right)\right\}^{1 / 2}$.

Thus, if we can find a finite number of points $q_{1}, \cdots, q_{m} \in R^{n}$ such that

$$
\bigcup_{i=1}^{m} \overline{I\left(q_{i}, \alpha\right)} \supset S^{n-1}(R) \quad \text { and } \quad \overline{I\left(q_{i}, \alpha\right)} \cap S^{n-1}(R) \neq \emptyset, i=1,2, \cdots, m
$$

and taking a point $\bar{p}_{i} \in \overline{I\left(q_{i}, \alpha\right)} \cap S^{n-1}(R)$ for each $i$, then, from the above fact, we have

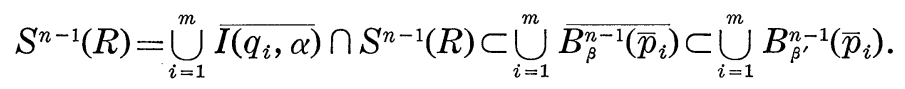

Hence, by means of an Euclidean geometric consideration, we can choose an $m$ with the above mentioned property such that

$$
m \leqq\left\{\begin{array}{lll}
\left(\left[\frac{2 R}{\alpha}\right]+1\right)^{n} & \text { if }\left[\frac{2 R}{\alpha}\right] \neq \frac{2 R}{\alpha} \\
{\left[\frac{2 R}{\alpha}\right]^{n}} & \text { if }\left[\frac{2 R}{\alpha}\right]=\frac{2 R}{\alpha} . & \text { q.e.d. }
\end{array}\right.
$$

Remark. The estimation of the number $m$ in the proof of Lemma 2 is very rough. We counted the number of points $q_{i} \in R^{n}$ such that $\overline{I\left(q_{i}, \alpha\right)} \cap S^{n-1}(R)=\emptyset$. When $\alpha$ is small with respect to $R$, then, for example, we can choose an upper bound of $m$ as

$$
m \leqq \begin{cases}\left\{2\left(\left[\frac{R}{\alpha}\right]+1\right)\right\}^{n}-\left\{2\left[\frac{R}{\sqrt{n} \alpha}\right]\right\}^{n} & \text { if }\left[\frac{R}{\alpha}\right] \neq \frac{R}{\alpha} \\
\left(2\left[\begin{array}{c}
R \\
\alpha
\end{array}\right]\right)^{n}-\left(2\left[\frac{R}{\sqrt{n} \alpha}\right]\right)^{n} & \text { if }\left[\begin{array}{c}
R \\
\alpha
\end{array}\right]=\frac{R}{\alpha} .\end{cases}
$$

And it will be possible to get a more sharp estimation.

Using this Lemma, we have

Proposition 2. Let $M$ be an n-dimensional compact Riemannian manifold with the diameter $d$ and the injectivity radius $\delta$, whose sectional curvature $K_{\sigma}$ satisfies $-b^{2} \leqq K_{\sigma} \leqq 0$ for some $b>0$. Then there exists a finite number of points $p_{1}, \cdots, p_{k} \in M$ such that

$$
\bigcup_{i=1}^{k} B_{\delta / 4}\left(p_{i}\right)=M \quad \text { with } \quad k \leqq\left(\left[\begin{array}{c}
4 d \\
\delta
\end{array}\right]+1\right)\left(\left[\frac{2 R}{\alpha}\right]+1\right)^{n}+1
$$

where $R$ and $\alpha$ are determined as in Lemma 2 by replacing $r$ and $s$ with $d$ and $\delta / 4$ respectively.

Proof. Let $\pi: \tilde{M} \rightarrow M$ be the universal covering of $M$ and $\pi$ its projection. Then as is well known, $\tilde{M}$ is diffeomorphic to an $n$-dimensional Euclidean space and each geodesic segment of $M$ is the shortest connection between its end points. Fix a point $p \in M$ and $\widetilde{p} \in\left\{\pi^{-1}(p)\right\}$. Clearly $B_{d}(\tilde{p})$ contains a fundamental domain $D$ of this covering i.e. $\pi: D \rightarrow M$ is one-to-one and $\pi: \bar{D} \rightarrow M$ is onto. Since $\delta$ is the injectivity radius of $M$, for each point $\tilde{q} \in \tilde{M}, \pi: B_{\delta / 4}(\tilde{q}) \rightarrow B_{\delta / 4}(\pi(\tilde{q}))$ is onto and isometric. So, if we can find a finite number of points $\tilde{p}_{1}, \cdots, \tilde{p}_{k} \in B_{d}(\tilde{p})$ satisfying $\bigcup_{i=1}^{k} B_{\delta / 4}\left(\tilde{p}_{i}\right) \supset \overline{B_{d}(\tilde{p})}$, then we have $\bigcup_{i=1}^{k} B_{\delta / 4}\left(\pi\left(\tilde{p}_{i}\right)\right)=M$. Let $H$ and 
$\bar{p} \in H$ be the space and the point as in Lemma 2. Let $l: T_{\tilde{p}}(H) \rightarrow T_{\tilde{p}}(\tilde{M})$ be an isometry. And consider a mapping

$$
\varphi:=\exp _{\tilde{p}} \cdot l \cdot \exp _{\tilde{p}}^{-1}: H \rightarrow \tilde{M} \text {. }
$$

For any point $\bar{q} \in B_{d}(\bar{p})$, let $\gamma:[0, \delta / 4] \rightarrow H$ be a geodesic starting from $\bar{q}$. Then, by Rauch's Comparison Theorem (see [2: Korollar p. 179]),

$$
L(\gamma) \geqq L(\varphi(\gamma))
$$

where $L$ denotes the length of a curve. So $\varphi\left(B_{\delta / 4}(\bar{q})\right) \subset B_{\delta / 4}(\varphi(\bar{q}))$ for any $\bar{q} \in B_{d}(\bar{p})$. Thus, if we find a finite number of points $\bar{p}_{1}, \cdots, \bar{p}_{k} \in H$ such that $\bigcup_{i=1}^{k} B_{\delta / 4}\left(\bar{p}_{i}\right) \supset \overline{B_{d}(\bar{p})}$, then, from the above facts, we have

$$
\left.\overline{B_{d}(\tilde{p})}=\overline{\varphi\left(B_{d}(\bar{p})\right.}\right) \subset \bigcup_{i=1}^{k} \varphi\left(B_{\delta / 4}\left(\bar{p}_{i}\right)\right) \subset \bigcup_{i=1}^{k} B_{\delta / 4}\left(\varphi\left(\bar{p}_{i}\right)\right) .
$$

And the existence of such points $\bar{p}_{i}$ is already shown in Lemma 2.

Summerising the above,

q.e.d.

Theorem. Let $M$ be an n-dimensional compact connected Riemannian manifold with non-positive sectional curvature $K_{\sigma} ;-b^{2}$ $\leqq K_{\sigma} \leqq 0$ for some $b>0$ and with Ricci curvature negative at some point. Let $d$ and $\delta$ be the diameter and the injectivity radius of $M$ respectively. Then

$$
\text { the order of } I(M) \leqq k^{k} \text {, }
$$

where $k$ is given by $k=\left(\left[\frac{4 d}{\delta}\right]+1\right)\left(\left[\frac{2 R}{\alpha}\right]+1\right)^{n}+1$ and $R, \alpha$ are determined with $\beta, \alpha$ as follows:

$$
\begin{gathered}
\cosh b \beta=\cosh \frac{b \delta}{4} / \cosh \frac{b \delta}{8}, \quad a=\tanh \frac{b d}{2} / b, \\
R=2 a /\left(1-a^{2} b^{2}\right), \quad \alpha=\left\{\frac{2 R^{2}}{n}\left(1-\cos \frac{\beta}{R}\right)\right\}^{1 / 2} .
\end{gathered}
$$

\section{References}

[1] T. Frankel: On Theorems of Hurewitz and Bochner. J. Math. Mech., 15, 373-377 (1966).

[2] D. Gromoll, W. Klingenberg, and W. Meyer: Riemannsche Geometrie im Grossen. Springer-Verlag (1968).

[3] H. C. Im Hof: Ü̉ber die Isometriegruppe einer kompacten Mannigfaltigkeiten negative Krummung. Comm. Math. Helv., 48, 14-30 (1973).

[4] H. Huber: Über die Isometriegruppe einer kompakten Mannigfaltigkeit mit negativer Krummung. Helv. Phys. Acta, 45, 277-288 (1972).

[5] H. B. Lawson and S. T. Yau: Compact manifolds of nonpositive curvature. J. Diff. Geom., 7, 211-228 (1972). 